ROCZNIKI HUMANISTYCZNE

Tom LXVIII, zeszyt 5 - 2020

DOI: http://dx.doi.org/10.18290/rh20685-6

HANS RICHARD BRITTNACHER

\title{
DIE ANDERE SHOAH: DER GENOZID AN DEN ,ZIGEUNERN` IN DER LITERATUR NACH 1989
}

\begin{abstract}
A b s tr a k t. Vorurteile gegen die Angehörigen der Romvölker, die immer noch als Zigeuner diskriminiert werden, prägen auch den erinnerungskulturellen Diskurs der Shoah. Der Genozid an den sog. Zigeuner wird geleugnet oder bagatellisiert. Dass es nur wenige Zeugnisse von Überlebenden gibt, erscheint in der Perspektive der Mehrheit als ästhetisches Unvermögen, Extremtraumatisierungen zu verarbeiten, während eher die Konventionen einer abweichenden Schamkultur den Betroffenen verbieten, sich darüber in der Sprache der Täter zu äußern. Erst die engagierte Arbeit von Bürgerrechtsbewegungen hat es Betroffenen möglich gemacht, in Zusammenarbeit mit Schriftstellern und Publizisten der Mehrheitskultur über den Genozid an den Roma zu schreiben.
\end{abstract}

Schlüsselwörter: Erinnerungskultur; Genozid; Holocaust; Gypsies; Roma; Sinti; Papusza; Colum McCann.

In José Giovannis Film Le Gitan (1975) spielt kein Geringerer als der französische Star Alain Delon mit herabhängendem Schnurrbart und traurigem Blick den Gauner Hugo Senart, einen ,Zigeunern', den die Korruption der französischen Behörden in die Kriminalität getrieben hat; jetzt raubt er Banken aus. Von der Polizei gehetzt findet er Zuflucht bei einem kultivierten Safeknacker, der ihm ein Dach überm Kopf und zur nächtlichen Lektüre ein Buch anbietet. Hugo ist dankbar, aber weist verlegen lächelnd das ihm angebotene Buch zurück und erbittet sich „eines mit Bildern.“,Zigeuner“, so lässt sich diese Szene verstehen, können nicht lesen, sie halten sich lieber an die Bilder. Auch in seinen anderen Szenen bestätigt der Film dem Zuschauer liebgewordene Vorurteile: sucht Hugo seinen Stamm auf, tanzen glutäugige junge Frauen in bunten Röcken ums Lagerfeuer, während die heranwachsenden

Prof. Dr. habil. HANS RichARD BRITTNACHER - Freie Universität Berlin, Fachbereich Philosophie und Geisteswissenschaften, Institut für Deutsche und Niederländische Philologie; Korrespondenzadresse: Habelschwerdter Allee 45, 14195 Berlin; E-Mail: brittnacher21@aol.com. ORCID: https://orcid.org/0000-0003-0282-2510. 
jungen Männer mit im Feuerschein blitzenden Zähnen sich im Messerkampf üben. Obwohl die Mehrheit der in Europa lebenden Angehörigen der Romvölker, ca. 10-12 Millionen (das sind mehr, als die Schweiz Bürger zählt), sesshaft ist, obwohl viele der als ,Zigeuner' diskriminierten Sinti mit Anzug und Krawatte zur Arbeit gehen, und obwohl die allermeisten Roma schreiben und rechnen können wie alle anderen auch - und wenn nicht, liegt dies eher an der Mehrheitsbevölkerung, die ihnen den Zugang zu Bildungseinrichtungen verwehrt oder erschwert - hält sich kaum ein Vorurteilskomplex so hartnäckig wie der von den ,Zigeunern' als unverbesserlichen Nomaden, als Kleinkriminellen, Vagabunden und eben: Analphabeten (Brittnacher).

Wer das glaubt, hat zumeist kein Problem, sich in einer Buchhandlung mit Anthologien zu versorgen, die ihm pittoreske Zigeunermärchen oder Zigeunerlieder anbieten, Operetten zu hören, in denen feurige ,Zigeunern' in der Puszta virtuos wie die Teufel auf der Geige musizieren, oder in der Oper hingerissen Carmens Bekenntnis zur freimütigen Liebe im Zigeunerstamm zu lauschen - es sind Heterostereotypen, Bilder aus der Außenperspektive, die unser Bild von den Roma bestimmen, seit Jahrhunderten gepflegt und solange weiterverbreitet, bis nahezu jeder sie glaubt. Gewiss gibt es auch Autoren, die es gut mit den Roma meinen, aber auch in ihren Texten spricht sich zumeist eine paternalistische Herablassung aus, der Wunsch, einem ,Volk ohne Stimme“ die eigene Stimme zu leihen. (Grass).

So lange es auch gedauert hat, den Holocaust in der deutschen Literatur $\mathrm{zu}$ thematisieren, so ungern er diskutiert wird, so überdrüssig viele seiner Erwähnung sind, so wird doch nur, wer nicht bei klarem Verstande ist, heute noch den Genozid an den Juden leugnen. Eine Bevölkerungsgruppe aber, die gleichfalls aus rassistischen Gründen flächendeckend erfasst, verfolgt, drangsaliert, deportiert, zwangssterilisiert und ermordet wurde, muss immer noch darauf warten, in der boomenden Erinnerungskultur einen angemessenen Platz einnehmen zu können - die ,Zigeuner'.

Unbefangen lässt sich der Begriff ,Zigeuner" nicht länger gebrauchen, aber problemlos ist auch der vom Zentralrat deutscher Sinti und Roma in Heidelberg angemahnte konsequente Gebrauch des Terminus Roma nicht, da dieser im Romanes, also der Sprache der Roma, nur soviel wie Mensch meint. Andere Gruppierungen wie die Lalleri oder die Litautikker lehnen diese Bezeichnung für sich $a b$, haben aber keine Einwände, mit dem von an-

\footnotetext{
${ }^{1}$ Im Folgenden wird weitgehend die Terminologie der Literatur übernommen, die in der Regel eben nicht von Sinti oder Roma, sondern von Zigeunern spricht. Allerdings sollten dabei die bedenklichen, xenophoben Implikationen des Begriffs deutlich werden.
} 
deren als diskriminierend empfundenen Label ,Zigeunern' bedacht zu werden. Auch die Sinti Allianz, erst in Köln, seit 2012 in Bergisch Gladbach, beispielsweise stört sich nicht an der Beibehaltung des Begriffs ,Zigeuner'. Es ist schwer zu entscheiden, welcher der beiden Verbände - der Zentralrat in Heidelberg oder die Sinti-Allianz in Bergisch Gladbach - mit größerer Autorität spricht, Aber keinesfalls ist es unstrittig, dass der Terminus zumeist diskriminierend gemeint und auch so empfunden wird.

Wie man sie auch nennen mag: auch ,Zigeuner' wurden Opfer des Genozids, des, so nennt es Dotschy Reinhardt, die Sängerin aus der Familie des Jazz-Gitarristen Djano Reinhart, „vergessenen Holocaust“ (Reinhardt). Die ,Zigeuner', die in Deutschland seit hunderten von Jahren ansässigen Sinti wurden in den Lagern gequält und systematisch ermordet, Roma aus den besetzten Ländern wurden Opfer von Massakern der Wehrmacht, bei Partisanenbekämpfungen oder bei Maßnahmen der Sicherheitspolizei. Wie viele Roma, Sinti und Jenische der nationalsozialistischen Vernichtung zum Opfer gefallen sind, ist umstritten. Seit der damalige Bundespräsident Roman Herzog von 500.000 Opfern sprach, hat sich diese Zahl als Richtwert durchgesetzt, einige Historiker behaupten, dass es erheblich weniger, etwa 100.000 seien, andere wollen die Zahl deutlich nach oben korrigiert sehen und sprechen von etwa zwei Millionen Opfern (Zimmermann, Rassenutopie und Genozid; Zimmermann, „Die nationalsozialistische“). Wenn die Zahl der vernichteten ,Zigeuner" also auch geringer ist als die der ermordeten Juden, kann man doch bei einer mutmaßlichen Opferzahl im mindestens sechsstelligen Bereich von einem mit systematischer eliminatorischer Energie betriebenen Genozid sprechen, der in der Konsequenz der Auschwitz-Logik steht, „die als rationales Projekt zur totalen Vernichtung ,minderwertiger' Rassen definiert werden kann." (Eder-Jordan 119) Inwieweit deshalb auch die Vernichtung der ,Zigeuner" unter den Oberbegriff Holocaust fällt oder ob dafür ein eigenständiger Begriff, etwa der des Porrajmos (Hancock, aber auch Tscherenkow und Laederich) gebraucht werden sollte, ist zwischen den Angehörigen von jüdischen und von Sinti- und Roma-Bürgerrechtsbewegungen z.T. heftig umstritten (Lewy; Wippermann). „They were not the chosen victim“, so heißt es bei Guenter Lewy, einem der renommiertesten amerikanischen Holocaust-Forscher, in Bezug auf die ,Zigeuner': Auserwählte Opfer seien nur die Juden gewesen (Lewy 203). Das Bewusstsein der Einzigartigkeit der Shoah drohe verlorenzugehen, wenn diese mit anderen Genoziden verglichen werde. Der Nobelpreisträger Elie Wiesel hat diese Überlegung in 
einer berühmten Bemerkung auf den Punkt gebracht: „Nicht alle Opfer [des Nationalsozialismus, H.R.B.] waren Juden, doch alle Juden waren Opfer.“

Dass der Völkermord an den Sinti und Roma noch weniger als der Holocaust - deutlich weniger, um es unmissverständlich zu sagen: nur an der Grenze zum Ephemeren - in der Literatur der Nachkriegsjahre reflektiert wurde (Brittnacher, „Erinnerungen“; Brittnacher, „Die Repräsentation“), verweist indes nicht auf die Sensibilität der deutschen Literatur für diese Differenzierungen, sondern nur auf die Zählebigkeit der deutschen antiziganistischen Tradition (Breger; Hille; Saul; Bogdal, Europa).

Während der Erinnerungsdiskurs im Fall der ermordeten Juden mittlerweile einen vitalen Bestandteil unseres kulturellen Selbstverständnisses darstellt, bleibt der Völkermord an den Roma nach wie vor ein nur selten thematisierter, zuweilen sogar verschwiegener Sachverhalt. Dass es vergleichsweise wenige Darstellungen des Lebens, Sterbens und Überlebens in den nationalsozialistischen Vernichtungslagern aus der Feder von überlebenden Roma gibt, heißt freilich nicht, dass Roma sich nicht erinnern oder nicht schreiben können, sondern dass es gute Gründe geben kann, das Erinnerte nicht mitteilen $\mathrm{zu}$ wollen. Gelegentlich findet sich in der Holocaustforschung die These, dass eine an oralen Traditionen orientierte Literatur der sprachlichen Artikulation eines Extremtraumas nicht gewachsen wäre, weil nur die hochgradige Verdichtungsarbeit und komplexe Polyphonie eines modernen literarischen Textes das Ungeheuerliche des industrialisierten Massenmordes ästhetisch angemessen vermitteln könne, aber noch in diesem Argument ist die alte Herablassung gegenüber einer literarischen Tradition spürbar, der unterstellt wird, mit ihren literarischen Mitteln nicht auf der Höhe der Zeit zu sein.

Philologische Evaluationen oraler Poesie werden durch den extemporierenden Charakter der Texte, die je nach Anlass oder Publikum ihre Gestalt wechseln können, erheblich beeinträchtigt. Eine neuere, durchaus repräsentative Abhandlung zur Mündlichkeit in der zeitgenössischen Literatur von Sinti und Roma entwickelt ihr Plädoyer für die Gleichrangigkeit oraler Poesie am Beispiel der Passagen mündlicher Rede von Romafiguren in zeitgenössischen Prosatexten und bestätigt so Vorbehalte gegen die Kompetenzen oraler Poesie. (Krumwiede-Steiner) In diesem Zusammenhang mag eine gattungstypologische Überlegung hilfreich sein: extreme Traumatisierungen sperren sich gewiss gegen die Sprache einer lebensgeschichtlichen Erzählung, Lyrik, Musik und bildende Kunst hingegen waren für viele Sinti und Roma durchaus gebräuchliche ästhetische Praktiken, ihre Erfahrungen, auch 
die in den Lagern erlebten, festzuhalten oder zu gestalten (Reinhardt 20). Vor allem aber sind es eher in der Kultur der Roma verbindliche Sprachregelungen des Respekts, der Diskretion und der Pietät, die mit der Pflicht des Chronisten zur ungenierten Wiedergabe des Schrecklichen kollidieren. Zudem schreibt, wer über den Mord an den Roma schreibt, möglicherweise auch in der Sprache der Täter. Er diskreditiert sich damit bei jenen, die nicht vergessen wollen, dass auch die Erlasse zu ihrer Vernichtung in der Sprache Goethes und Schillers verfasst waren - ein immer wieder auch von jüdischen Autoren reflektierter Sachverhalt. So haben denn auch Roma, die literarisch Zeugnis davon gegeben haben, was ihnen und ihrem Volk angetan wurden, nicht selten durch die Veröffentlichung ihrer Erinnerungen den Konflikt mit einer Mehrheit ihres Volkes riskiert, die auf strikter Verschwiegenheit bestand, weil sie ihre erschütternden Erinnerungen an Exekutionen und Folter nicht mit der Generation der Täter und deren Nachkommen teilen wollte. Für viele mittel- und osteuropäische Roma, die durch die rigorose Assimilationspolitik der sozialistischen Republiken zu oft ungewollter Alphabetisierung gezwungen wurden, war es, wie die Prager Indologin und Begründerin der tschechischen Romistik, Milena Hübschmanova schreibt, eine unglaubliche Entdeckung, dass sich im Romanes auch Gedichte und Erzählungen schreiben ließen (Hübschmanova). Der kühlen wissenschaftlichen Beobachtung steht freilich das in Jahrhunderten der Unterdrückung gewachsene Misstrauen von Roma gegenüber, die an Wohltaten der Obrigkeit nicht glauben wollen. Der irische Autor Colum McCann hat 2006 unter dem Titel Zoli einen erfolgreichen Roman über das Schicksal einer slowenischen Romni vorgelegt, die in der politischen Aufbruchstimmung der 50er Jahre des letzten Jahrhunderts beginnt, in der Sprache ihrer vormaligen Verfolger die Geschichte ihres Volkes und ihre Lieder aufzuzeichnen, was ihr die Ächtung durch ihr Volk einträgt. Die fiktive Gestalt der Zoli Lackova ist eng an das Schicksal der polnischen Sängerin und Lyrikerin Papusza angelehnt.

Dass es lange dauerte, bis es überhaupt zu einem Versuch der literarischen Aufarbeitung des Vergangenen kam, hat zum einen mit der Notwendigkeit oder dem Wunsch vieler Roma zu tun, im Land der Täter weiterzuleben, zum anderen mit psychischen Widerständen, das erlebte Grauen zu vergegenwärtigen und die erlittenen Demütigungen mitzuteilen. Eine initiale Bedeutung kommt einem Hungerstreik von Bürgerrechtsaktivisten im vormaligen Konzentrationslager Dachau zu, der auf die fatale Lage der Sinti in Deutschland aufmerksam machte, zur Bildung einer Bürgerrechtsbewegung führte und eine Reihe von Überlebenden dazu brachte, ihre Erinnerungen in 
dem Sammelband ... weggekommen. Berichte und Zeugnisse von Sinti, die die NS-Verfolgung überlebt haben (2000), niederzulegen. In Interviews mit Ilona und Reinhard Lagrene, die teils in romanes, teils in deutsch geführt und übersetzt und zusätzlich weiter redaktionell bearbeitet wurden, artikulieren die Interviewten, ,wie unverzichtbar und wichtig für die Überlebenden und ihre Nachkommen - das Zeugnis geben wie das Zeugnis-nehmen ist. Zeugnis nehmen ist das, was den nachfolgenden Generationen zu tun bleibt, als Verpflichtung unabhängig davon, zu welcher Volksgruppe oder Minderheit wir gehören." (Strauß 12) Ihren Durchbruch erlebte die Erinnerungsliteratur von Roma durch Philomena Franz und durch Ceija Stojka.

Philomena Franz hat in ihrem zweiten Buch Zwischen Liebe und Hass, Ein Zigeunerleben (1985) beschrieben, wie der unbeschwerten Jugend das NS-Regime mit Schulverbot, Zwangsarbeit und die Deportation von Familienmitgliedern folgte. Sie schildert ihre Zeit in Auschwitz sowie das „Weiterleben nach dem Nullpunkt." Sie sieht sich, wie ihre Schriften gerade auch in den Neuauflagen bezeugen, als eine ,Zigeunerin': „Ich habe dieses Buch als Zigeunerin geschrieben. Als Zigeunerin vom Stamm der Sinti“ (Franz 10). Sie geht davon aus, dass sie damit einer Bevölkerungsgruppe angehört, die grundlegend anders ist als die Mehreitsbevölkerung: „Wir denken anders. Wir fühlen anders." Damit formuliert sie ein Selbstverständnis, das sich mit überkommenen mehrheitsgesellschaftlichen Konstruktionen von einer Andersartigkeit des „zigeunerischen Wesens“ deckt, aber heute von vielen Roma abgelehnt wird. Ceija Stojka, die österreichische Künstlerin, beschrieb unter dem Titel Wir leben im Verborgenen. Erinnerungen einer RomZigeunerin (1988) ihre Erinnerungen an den Aufenthalt in drei Konzentrationslagern. In einem Gespräch mit der Journalistin Katrin Berger weist sie darauf hin, welche Widerstände ihrem Projekt auch von den ihr Nahestehenden entgegengebracht wurden, wagte es hier doch eine Frau in einer stark patriarchalisch geprägten Kultur, Zeugnis für das Leiden ihres Volkes abzulegen, und das in einer Sprache, die nicht die ihre war und zudem für ein Publikum aus gadsche, aus Nicht-Zigeunern also (vgl. Stojka, „Du darfst keine andere sein“ 97). Krista Hauser berichtet im Vorwort zu einer Gedichtsammlung von Ceija Stojka vom ambivalenten Verhältnis der Autorin zur sprachlichen Vergegenwärtigung des Erinnerten: „Nach ihren Büchern Wir leben im Verborgenen und Reisende auf dieser Welt hat sie weiterhin notiert, was sie bewegt. ,Ich schreibe, obwohl ich es nicht kann.' Diesen authentischen Satz [...] wiederholte sie oft. Lachend, nachdenklich, manchmal traurig. Nicht können, das heißt Schreiben ohne Duden-Deutsch. Sie ist im 
Wienerischen, in der österreichischen Umgangssprache zu Hause, träumt und denkt meist in ihrer Mutter- und Vatersprache, dem Romanes [...]" (Hauser 8). Auch Ceja Stojkas Brüder sollten Jahre später schließlich literarisch produktiv werden und den Bann des Schweigens brechen: Der Maler Karl Stojka veröffentlichte unter dem Titel Auf der ganzen Welt zu Hause (1994) seine Autobiographie und Mongo Stojka, ein Musiker, brachte seine Erinnerungen unter dem Titel Papierene Kinder. Glück, Zerstörung und Neubeginn einer Roma-Familie in Österreich (2000) zu Papier. Beides sind Werke, in denen sich im Wechsel lakonischer und düsterer Passagen mit privaten, oft auch schelmischen Erinnerungen die mündliche Erzähltradition der Lovara, der nomadisierenden Pferdehändler, von denen die Stojkas abstammen, abbildet. Otto Rosenberg, langjähriges Mitglied im Zentralrat deutscher Sinti und Roma und Vorsitzender des Berliner Landesverbandes, hat jahrelang die quälenden Erinnerungen an seine Kindheit und Jugend als Sinto im Berlin der 30er und 40er Jahre und als Zwangsarbeiter in nationalsozialistischen Konzentrationslagern, wo der größte Teil seiner Familie den Tod fand, mit sich herumgeschleppt, bis er es endlich über sich brachte, sie Tonbändern anzuvertrauen, die Ulrich Enzensberger - wie er betont: originalgetreu - wiedergab, um den Gestus der oralen Tradition, das Stocken und Verstummen, aber auch den eigentümlichen Bilderreichtum dieser Sprache, an der das Erlebte und Erlittene unlöslich wie Pech festhaftet, nicht zu beeinträchtigen. Auch in den Lebenserinnerungen des Sinto Hugo Höllenreiner Denk nicht, wir bleiben hier (2005), die Anja Tuckermann aufzeichnete, wobei ihre sorgfältige graphische Gestaltung zwischen dem originalen Wortlaut und überarbeiteten Passagen unterschied, bringt sich immer wieder die schubweise und schmerzhaft aufbrechende Erinnerung an das Lagertrauma zur Geltung. Dass die Kultur der Romvölker oft auf orale Traditionen zurückgreift, macht sie keineswegs sprachlos.

Klaus Michael Bogdal, gewiss einer der besten Kenner der Materie, stellt in seinem Nachwort zu der Anthologie Die Morgendämmerung der Worte die Erinnerungen Höllenreiners Ruth Klügers Weiter leben an die Seite. „Höllenreiner erzählt wie die meisten überlebenden Sinti und Roma zögernd und gegen große innere Widerstände, weil das Reden ihn erneut seinen Erlebnissen ausliefert und niemand in der Lage ist, sie aufzufangen, wenn die Erinnerungen einbrechen. [...] In den Lebenserinnerungen geht es immer wieder darum, gegen Lügen und Verdrehungen die Wahrheit des Selbsterlebten zu setzen und die persönliche Erfahrung als einen Teil der Geschichte ins Spiel zu bringen. $\mathrm{Zu}$ verspüren ist ebenso der Wunsch, sich aus eigener Kraft 
von stummen, überwältigten Opfern zu einem selbstbestimmten Subjekt zu erheben“ (Bogdal, „Symbolische Gewalt“ 359).

Das mag, wenn es denn gelingt, gewiss wünschenswert sein - aber den intrikaten Aspekt des Sprechens der Zigeuner über die Shoah übergeht auch Bogdal: Ceija Stoka entwickelt ihre Erinnerungen an den Aufenthalt in Konzentrationslagern in einem Gespräch mit der Journalistin und Regisseurin Katrin Berger, Otto Rosenberg vertraut seine Erinnerungen über die Zwangsarbeit in nationalsozialistischen Konzentrationslagern und den Tod seiner Familienangehörigen Tonbändern an, die Ulrich Enzensberger, der Schriftsteller, zu Papier brachte, und Hugo Höllenreiners Erinnerungen hat Anja Tuckermann in Gesprächen, die sich über ein Jahr hinzogen, aufgezeichnet. Immer sind es andere, die das Sprechen der ,Zigeuner' der Schriftkultur, also der literalen Konvention der Mehrheitsgesellschaft anpassen. Wilhelm Solms ging so weit, der Autorin Anja Tuckermann vorzuwerfen, sich auf Kosten eines Sinto profiliert zu haben, weil die Erinnerungen Höllenreiners bibliographisch unter ihrem Namen erscheinen (Solms $13 \mathrm{f}$.). Das geht gewiss zu weit: Die Integrität von so verdienten Publizisten und Schriftstellern wie Katrin Berger, Ulrich Enzensberger und Anja Tuckermann steht außer Frage, wohl aber leidet die Authentizität von Erinnerungen, wenn sie sich einer doppelten Urheberschaft verdanken, der mündlich erinnerten der einen und der von anderen verschriftlichten. Tuckermann gesteht ihre radaktionelle Bearbeitung von Höllenreiners unter Qualen freigesetzten Erinnerungen im Interesse angemessen literarischer Wirkung, die für den Betroffenen zudem kathartisch sein könne, durchaus ein. „Ich beschloss, seine Geschichte in der dritten Person zu schreiben, angelehnt an seine Sprache neu zu formulieren, weil grauenvolle Erlebnisse sich nicht geordnet erzählen lassen, die Erinnerung nicht gradlinig verläuft. Oft reißt ein Satz ab, weil es zu schlimm ist, weiterzusprechen; oder in dem Augenblick die Erinnerung zu ertragen“ (Tuckermann 290). Mit den - von ihr in Schriftsprache übersetzten - Worten Höllenreiners aktiviert sie eine verschüttete Erinnerung an unendlich viele erfrorene Kinderleichen am Wegesrand: „Ich habe nichts mehr davon gewusst, bis heute nicht. Heute Nacht habe ich plötzlich alles wieder vor mir gesehen. Die Kinder müssen schon eine Weile da gelegen haben, starr. Ich hatte so einen Druck im Kopf und jetzt weiß ich es wieder. Ich bin jetzt so frei im Kopf. Ich könnte heulen vor Freude“ (Tuckermann 178). Den behaupteten kathartischen Gewinn dieser ,gelungenen' Erinnerungsarbeit schränkt der zum Sprechen und Erinnern Gebrachte an anderen Stellen ein, aber auch dies in den Worten seiner Protokollantin. „Gestern ist es mir nicht 
gut gegangen. Heute schon den ganzen Tag. Magenweh, Kopfweh und Gedanken, Gedanken. Je mehr ich erzähle, ich merke es doch an mir, je mehr das rauskommt, desto besser wird's mir dann. [...] Richtig frei bin ich trotzdem nie" (Tuckermann 287).

Während in Westeuropa, überhaupt im gesamten Bereich der Romania, aber auch in Mittel- und Osteuropa mittlerweile eine vitale Literatur in Romanes, also der Sprache der Roma, bzw. in einer der in Europa gesprochenen Romanes-Varianten entsteht und damit endlich auch die Selbstdarstellung von Roma-Künstlern und Literaten an die Stelle der über sie verbreiteten Klischees tritt, ist es offenbar das Schicksal der deutschsprachigen Literatur der Roma, auch heute, zu einer Zeit, da es kaum noch lebende Zeugen der Shoah gibt, am Zivilisationsbruch von Auschwitz nicht vorbeischreiben zu können. Marianne Rosenberg, eine in Deutschland bekannte und populäre Schlagersängerin, die Tochter von Otto Rosenberg, kommt in ihrer Autobiographie Kokolores (2006) auch auf die Nöte des von seinen Erinnerungen gequälten Vaters zu sprechen, der nachts laut weinte und nach den Toten rief. Und auch die Jazz-Sängerin Dotschy Reinhardt aus der weltberühmten Dynastie der Sinti-Jazzer Django und Schnuckenack Reinhardt entwickelt in ihrem Text Gypsy: Die Geschichte einer großen Sinti-Familie (2008) ihr Selbstverständnis, aber auch ihr Selbstbewusstsein gerade aus dem trotzigen Bekenntnis zu ihrem Außenseiterstatus und über die Reflexion der Verfolgungsgeschichte, der ihre Familie seit je ausgesetzt war. Angesichts der Leidensgeschichte der Roma kann Dotschy Reinhardts strikte Abweisung eines Assimilationsverlangens nicht verwundern - wenn auch, wie im östlichen und westlichen Ausland, die literarische Zukunft vielleicht eher einer hybriden Kultur gehört. ${ }^{2}$

\section{ZITIERTE WERKE}

\section{QUELLEN}

Die Morgendämmerung der Worte. Poesie-Atlas der Roma und Sinti, edited by Wilfried Ihrig and Ulrich Janetzki, Die andere Bibliothek, 2018.

Franz, Philomena. Zwischen Liebe und Hass, Ein Zigeunerleben. Herder, 1985 (1992).

Grass, Günter. Ohne Stimme. Reden zugunsten des Volkes der Sinti und Roma. Steidel, 2001.

\footnotetext{
${ }^{2}$ Für eine erste deutschsprachige Bestandsaufnahme dieser Tradition siehe Julia Blandfort und Marina M. Hertrampf, editors, Grenzerfahrungen - Roma-Literaturen in der Romania. LIT Verlag, 2011.
} 
Hancocks, Ian. We are the Romani people / Ame sam e Rromane džene. University of Herfordshire Press, 2002.

Le Gitan. Directed by José Giovanni, screenplay by José Giovanni, production: Alain Delon and Raymond Danon, performances: Alain Delon, Paul Meurisse, and Annie Girardot, Adel Productions, Lira Films, Mondial Televisione Film, 1975.

McCann, Colum. Zoli. Rowohlt, 2007.

Reinhart, Dotschy. Gypsy. Die Geschichte einer großen Sinti-Familie. Scherz, 2008.

Rosenberg, Marianne. Kokolores. Autobiographie. List, Ullstein, 2006.

Rosenberg, Otto. Das Brennglas. Aufgezeichnet von Ulrich Enzensberger. Wagenbach, 2012.

Stojka, Ceija. Wir leben im Verborgenen. Erinnerungen einer Rom-Zigeunerin, edited by Katrin Berger, Picus, 1989.

Stojka, Ceija. "Du darfst keine andere sein. Ceija Stojka im Gespräch mit Karin Berger." Stojka Ceija. Wir leben im Verborgenen. Erinnerungen einer Rom-Zigeunerin, edited by Katrin Berger, 1989a, pp. 97-155.

Stojka, Ceja. Meine Wahl zu schreiben - ich kann es nicht. O fallo de isgiri-me tschischanaf les. Gedichte (Romanes/deustch) und Bilder. EY3, 2003.

Stojka, Karl, and Reinhard Pohanka. Auf der ganzen Welt zu Hause. Das Leben und Wandern des Zigeuners Karl Stojka. Picus, 1994.

Stojka, Mongo. Papierene Kinder. Glück, Zerstörung und Neubeginn einer Roma-Familie in Österreich. Hanser, 2008.

Strauß, Daniel, editor. ... weggekommen. Berichte und Zeugnisse von Sinti, die die NS-Verfolgung überlebt haben. Philo, 2002.

Tuckermann, Anja. "Denk nicht, wir bleiben hier". Die Lebensgeschichte des Sinto Hugo Höllenreiner. (2005). DTV, 2018.

\section{FORSCHUNG}

"Zigeuner." Enzyklopädie des Holocaust. Die Verfolgung und Ermordung der europäischen Juden, edited by Eberhard Jäckel, Peter Longerich, Julius H. Schoeps. Band 3. Argon Verlag, 1998, pp. 1630-1634.

Blandfort, Julia, and Marina M. Hertrampf, editors. Grenzerfahrungen-Roma-Literaturen in der Romania. LIT Verlag, 2011.

Bogdal, Klaus-Michael. Europa erfindet die Zigeuner. Eine Geschichte von Faszination und Verachtung. Suhrkamp, 2011.

Bogdal, Klaus-Michael. "Symbolische Gewalt. Die Literatur und die ,Zigeuner"”. Nachwort zu: Die Morgendämmerung der Worte. Poesie-Atlas der Roma und Sinti, edited by Wilfried Ihrig and Ulrich Janetzki, Die andere Bibliothek, 2018, pp. 356-374.

Breger, Claudia. Ortlosigkeit des Fremden. ,Zigeunerinnen ' und ,Zigeuner' in der deutschsprachigen Literatur um 1800. Böhlau, 1998.

Brittnacher, Hans Richard. "Die Repräsentation des Genozids an Sinti und Roma in der deutschen Nachkriegsliteratur". Akten des XII. Internationalen Germanistenkongresses in Warschau, Bd.6: Vielheit und Einheit der Germanistik weltweit, edited by Franciszek Grucza, Peter Lang Verlag, 2013, pp. 227-236. 
Brittnacher, Hans Richard. "Erinnerungen an das Undenkbare: Der Porrajoms in der Literatur von und über ,Zigeuner". Geschichte und Gedächtnis in der Literatur vom 19. bis 21. Jahrhundert, edited by Janusz Golec and Irmela von der Lühe, Peter Lang Verlag, 2010, pp. 215-228.

Brittnacher, Hans Richard. Leben auf der Grenze. Klischee und Faszination des Zigeunerbildes in Literatur und Kunst. Wallstein, 2012.

Eder-Jordan, Beate. "Die nationalsozialistische Rassen- und Vernichtungspolitik im Spiegel der Literatur der Roma und Sinti”. Der nationalsozialistische Genozid an den Roma Osteuropas. Geschichte und künstlerische Verarbeitung, edited by Felicitas Fischer von Weikersthal, Christoph Garstka, Urs Heftrich and Heinz-Dietrich Löwe, Böhlau, 2008. 115167.

Hauser, Christa. "Ceija Stojka, eine Begegnung." Meine Wahl zu schreiben - ich kann es nicht. O fallo de isgiri - me tschischanaf les. Gedichte (Romanes/deustch) und Bilder. EY3, 2003 pp. 6-9.

Hille, Almut. Identitätskonstruktionen. Die Zigeunerin in der deutschen Literatur des 20. Jahrhunderts, Königshausen \& Neumann, 2005.

Hübschmanova, Milena. "Birth of Romani Literature in Czechoslowakia. Social and Political background." Cahier de Littérature orale, vol. 30, 1991, pp. 91-97.

Krumwiede-Steiner, Franziska. Formen und Funktionen von Mündlichkeit in der Gegenwartsliteratur der ,, Sinti und Roma.” LIT Verlag, 2017.

Lewy, Gunter. "Himmler and the ,Racial Pure Gypsies"”. Journal of Contemporary History, vol. 34, Heft 2, 1999, pp. 201-214.

Reinhardt, Dotschy. "Vorwort." Die Morgendämmerung der Worte. Poesie-Atlas der Roma und Sinti, edited by Wilfried Ihrig and Ulrich Janetzki, Die andere Bibliothek, 2018, pp. 14-24.

Saul, Nicholas. Gypsies and Orientalism in German Literature and Anthropology of the Long Nineteenth Century. Legenda, 2007.

Solms, Wilhelm. "Tuckermanns Aneignung von Höllenreiners Lebensgeschichte." Antiziganismusforschung, vol. 1, 2006.

Tscherenkow, Lev, and Stéphane Laederich. The Roma. Otherwise known as Gypsies, Gitanos, Tsiganes, Tigani, Cingene, Zigeuner, Bohémiens, Travellers, Fahrende etc. Band 1-2. Schwabe, 2004.

Wippermann, Wolfgang. ,Auserwählte Opfer?' Shoah und Porrajmos im Vergleich: eine Kontroverse. Frank \& Timme, 2005.

Zimmermann, Michael. "Die nationalsozialistische Verfolgung der Zigeuner. Ein Überblick". Sinti, Roma, Gypsies. Sprache - Geschichte - Gegenwart, edited by Yaron Matras, Hans Winterberg, and Michael Zimmermann. Metropol, 2003, pp. 115-153.

Zimmermann, Michael. Rassenutopie und Genozid. Die nationalsozialistische Lösung der Zigeunerfrage. Christians, 1996. 


\section{INNA ZAGŁADA: LUDOBÓJSTWO 'CYGANÓW' W LITERATURZE PO ROKU 1989}

Streszczenie

Uprzedzenia wobec narodów romskich, które ciągle jeszcze w sposób dyskryminujący nazywa się Cyganami, wpływają na kulturowy dyskurs pamięci o Szoah. Ludobójstwu tzw. Cyganów zaprzecza się bądź je bagatelizuje. Jako że istnieje niewiele świadectw tych, którzy przeżyli, z perspektywy większości (kultury dominującej: tu niemieckiej) uporanie się z traumą wydaje się być estetycznie niemożliwe, $\mathrm{z}$ drugiej zaś strony reguła kultury wstydu zabrania osobom dotkniętym ową traumą mówić o niej w języku sprawców. Dopiero inicjatywa ruchów obywatelskich oraz współpraca z pisarzami i publicystami kultury dominującej otworzyła Romom możliwość literackiego zmierzenia się z ludobójstwem.

Słowa kluczowe: kultura pamięci; ludobójstwo; Holocaust; Cyganie; Romowie; Sinti; Papusza; Colum McCann.

\section{THE OTHER SHOAH: THE GENOCIDE ON THE 'GYPSIES' IN LITERATURE AFTER 1989}

\section{S u m m a r y}

Prejudices against the Romani people, who are still often discriminatorily called Zigeuner, gypsies, gitanes or gitanos, still have an influence on the cultural memory discourse of the Shoah. The genocide of the Romani is either denied or trivialised. The fact that there are hardly any autobiographies of Romani survivors results from an inability to process extreme trauma, while the conventions of the people concerned prohibits them from speaking out about their trauma in the language of the perpetrators. It is thanks to the committed work of civil rights movements that Romani victims could collaborate with writers and publishers to finally tell the majority cultures of their suffering and of the genocide of the Romani people.

Key words: Cultural memory discourse; genocide; Holocaust; Roma; Sinti; Papusza; Colum McCann. 